\title{
Age Dependence of Gadolinium Leakage from the Cortical Veins into the Cerebrospinal Fluid Assessed with Whole Brain 3D-real Inversion Recovery MR Imaging
}

\author{
Shinji Naganawa*, Toshiki Nakane, Hisashi Kawai, and Toshiaki Taoka
}

\begin{abstract}
Purpose: It has been reported that intravenously administered gadolinium-based contrast agents (IV-GBCAs) leak into the cerebrospinal fluid (CSF) even in healthy subjects. The purpose of this study was to evaluate GBCA leakage from the cortical veins in patients with delayed imaging after IV-GBCA.
\end{abstract}

\begin{abstract}
Materials and Methods: There are two parts of retrospective study. In the first part, we reviewed six patients with suspected endolymphatic hydrops (EH) who received a single dose of IV-GBCA (37-58 years old). The 3D-real inversion recovery images were obtained prior to the contrast administration as well as $5 \mathrm{~min}$ and $4 \mathrm{~h}$ after IV-GBCA. Leakage from the cortical veins to the CSF was graded as positive if enhancement around the cortical veins at $5 \mathrm{~min}$ was observed and had further spread into the CSF at $4 \mathrm{~h}$ after IV-GBCA. In the second part of this study, we reviewed 21 patients with suspected EH (17-69 years old). Images were obtained only at $4 \mathrm{~h}$ after IV-GBCA. The number of slices (NOS) with a positive GBCA leakage from the cortical veins was counted. The correlation of the NOS with age, gender, and degree of EH was evaluated by Spearman's rank correlation coefficient.
\end{abstract}

Results: In the first part of the study, the GBCA leakage from the cortical veins was positive in all patients. In the second part of the study, the GBCA leakage from the cortical veins was seen in all older patients (above 37 years old), but not in the five younger patients (younger than 37 years old). The NOS correlated significantly only with age $(r=0.755, P<0.01)$, but not with gender or degree of $\mathrm{EH}$.

Conclusion: IV-GBCA leaks from the cortical veins into the surrounding CSF. The leakiness of the cortical veins significantly correlated with age, but not with gender or degree of $\mathrm{EH}$.

Keywords: cortical vein, gadolinium, glymphatic system, magnetic resonance imaging

\section{Introduction}

We have previously reported that intravenously administered gadolinium-based contrast agents (IV-GBCAs) leak into the cerebrospinal fluid (CSF) and perivascular space (PVS) even in healthy subjects without blood-brain barrier (BBB) disruption. ${ }^{1,2}$ GBCAs in the CSF can enter brain parenchyma, probably through the PVS in humans ${ }^{3-5}$ as well as animals. ${ }^{6}$ This "glymphatic" route is speculated to be the path by which GBCA enters the brain, and ultimately accumulates. ${ }^{6-8}$ The leakage points for GBCAs into the CSF have been suggested to include the peripheral part of the cranial nerves, the

Department of Radiology, Nagoya University Graduate School of Medicine, 65 Tsurumai-cho, Shouwa-ku, Nagoya, Aichi 466-8550, Japan

*Corresponding author, Phone: +81-52-744-2327, Fax: +81-52-744-2335, E-mail: naganawa@med.nagoya-u.ac.jp

C2018 Japanese Society for Magnetic Resonance in Medicine

This work is licensed under a Creative Commons Attribution-NonCommercialNoDerivatives International License.

Received: April 5, 2018 | Accepted: September 7, 2018 circumventricular organs (CVOs) and the spinal ganglions. ${ }^{1,2,9}$ In our clinical practice, we routinely obtain heavily $\mathrm{T}_{2}$-weighted 3D-fluid-attenuated inversion recovery (FLAIR) images for the evaluation of endolymphatic hydrops (EH) of the inner ear at $4 \mathrm{~h}$ after a single dose of IV-GBCA. ${ }^{10-15}$ Subsequently, we noticed that the signal intensity of the CSF around some of the cortical veins, such as the vein of Labbe, sometimes increased selectively. In a previous study, the axial imaging slab with multiple averaging for the evaluation of EH was centered on the inner ear and only covered the inferior half of the brain including the vein of Labbe. An improved hybrid of reversed image of positive endolymph signal and native image of positive perilymph signal (i-HYDROPS) technique utilizing a longer repetition time has a higher sensitivity to low concentrations of GBCA in fluid. ${ }^{11}$ Three-dimensional real inversion recovery (3D-real IR) imaging using a longer repetition time similar to the i-HYDROPS ${ }^{11}$ technique was reported to be useful for the evaluation of $\mathrm{EH}$ by avoiding the risk of paradoxical signal decrease by GBCAs and artifacts from misregistered 
subtraction images. ${ }^{16-18}$ Whole brain 3D-real IR imaging with a single excitation can obtain a similar signal-to-noise ratio to that obtained by a limited slab 3D-real IR image with multiple excitations. Therefore, we now routinely obtain whole brain 3D-real IR images for the evaluation of EH.

The purpose of this study was to retrospectively evaluate the leak of IV-GBCA from the cortical veins into the CSF space by reviewing whole brain 3D-real IR images in subjects that received a 3D-real IR scan for the evaluation of EH. We also evaluated the effect of age and gender, as well as the degree of $\mathrm{EH}$ on the leakiness of the cortical veins.

\section{Materials and Methods}

This retrospective study consisted of two parts. In the first part, the leakage of IV-GBCA from the cortical veins into the CSF space to be confirmed, we reviewed scans from patients who received pre- and post-contrast enhanced 3D-real IR imaging. The detailed scan parameters are shown in Table 1. The scan time was $10 \mathrm{~min}$. The images were obtained using a 3T MR scanner (Skyra; Siemens, Erlangen, Germany) at pre- and immediately after $(5 \mathrm{~min})$ and $4 \mathrm{~h}$ after IV-GBCA in six patients with a suspicion of EH (37-58 years old, median 55, three men). Subtracted images of 5 min minus pre-contrast and $4 \mathrm{~h}$ minus pre-contrast were generated on the scanner. For image evaluation, a positive enhancement was considered as fulfilling the two conditions below in multiple locations.

1. Linear enhancement along the cortical vein in the subtracted images acquired at $5 \mathrm{~min}$ post IV-GBCA.

2. A further spread of the linear enhancement along the cortical vein into the CSF space around the veins in the subtracted images acquired at $4 \mathrm{~h}$ post IV-GBCA.

In the second part of the study, we reviewed 21 patients with suspected EH who received IV-GBCA (17-69 years old, median 49, six men). The images were acquired on the same 3T MR scanner and using identical scan parameters as the first part of this study. In this second part, the images were obtained only at $4 \mathrm{~h}$ after IV-GBCA. More recently, we have not acquired the scans at pre- and immediately after IVGBCA, because we have confirmed that images at $4 \mathrm{~h}$ are sufficient to evaluate the presence of EH. In this part of the study, six axial slices at $20 \mathrm{~mm}$ interval were selected out of the 256 slices. The six slices were selected every $20 \mathrm{~mm}$ from the vertex. The images were evaluated on a PACS viewer (RapideyeCore, Cannon Medical Systems, Tokyo, Japan). The display condition was set as constant with a window width of 80 and a window level of 10 . Two experienced neuroradiologists (S.N. and T.T.) independently counted the number of slices (NOS) with positive GBCA leakage from the cortical veins into the surrounding CSF. The kappa statistic was calculated to evaluate the inter-observer variability. Consensus was obtained after discussion between the two

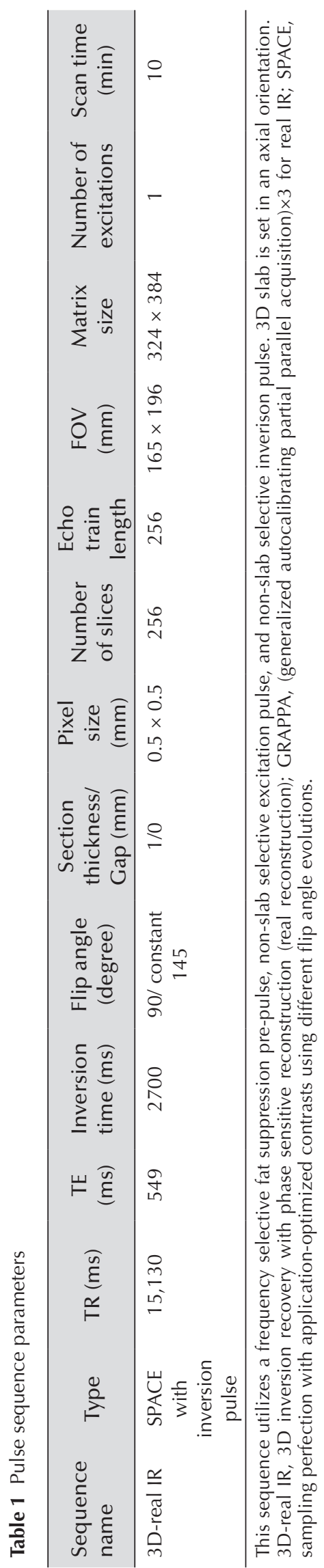


observers for any discrepancies. Correlations of the NOS with age, gender, and the degree of EH in the cochlea and the vestibule was evaluated by Spearman's rank correlation coefficient. For the EH grading, the previously proposed score (0: none, 1: mild, 2: severe) was used. ${ }^{19}$ The EH grading had been performed previously by an experienced neuroradiologist (S.N.) and was obtained from the diagnostic report. If there were no slices with a positive enhancement for a patient, all slices were carefully reviewed to confirm that there were no leakage points on the non-selected slices. The number of patients with a positive enhancement out of 21 patients at each slice level was compared among the slices by a one factor ANOVA with Bonferroni correction.

All patients in both parts of this study had an estimated glomerular filtration rate (eGFR) of more than $60 \mathrm{ml} / \mathrm{min} /$ $1.73 \mathrm{~m}^{2}$. All patients received only a single dose $(0.1 \mathrm{mmol} / \mathrm{kg})$ of IV administered gadobutrol (Gadovist; Bayer Pharmaceuticals, Osaka, Japan). The ethical committee of our institution approved this retrospective study with a waiver of consent from the patients. We used $5 \%$ as a threshold to determine statistical significance. The software $\mathrm{R}$ (version 3.3.2, $\mathrm{R}$ Foundation for Statistical Computing, Vienna, Austria, https:// www.R-project.org/) was used for the statistical analyses.

\section{Results}

In the first part of this study, the GBCA leakage from the cortical veins into the surrounding CSF was positive in all patients. (Fig. 1) The linear enhancement along the cortical veins was apparent in the images obtained at $5 \mathrm{~min}$ after
IV-GBCA and the enhancement spread into the CSF space in multiple sites.

In the second part of this study, there was only one slice with a discrepancy between the two observers out of 126 $(6 \times 21)$ slices $(k$-value $=0.984)$. The GBCA leakage from the cortical veins into the surrounding CSF was seen in all older patients (37 years and above), but not in the five younger patients (under 37 years: 16, 17, 23, 29, and 36 years old) (Figs. 2 and 3). In these younger patients, all 256 slices were reviewed to confirm the absence of the GBCA leakage from the cortical veins and there was no slice with positive GBCA leakage in all five young patients.

The NOS significantly correlated only with age $(r=0.755$, $P<0.01$ ) (Fig. 4). Neither the significant correlation was observed between the NOS and gender, nor between the NOS and the degree of EH. The number of patients with a positive signal enhancement out of 21 patients at each slice level was significantly higher at the most vertical slice compared to the third slice from the vertex and the most basal slice $(P<0.05)$. There was also a significant difference between the second slice from the vertex and most basal slice $(P<0.05)$ (Table 2).

\section{Discussion}

Recently, it has been reported that IV-GBCA leaks to the CSF in humans and animals without BBB disruption even in cases with normal renal function. ${ }^{1,2,9,10,14,20,21}$ Multiple leak points such as the anterior eye segment, the peripheral part of the various cranial nerves, and the circumventricular organs
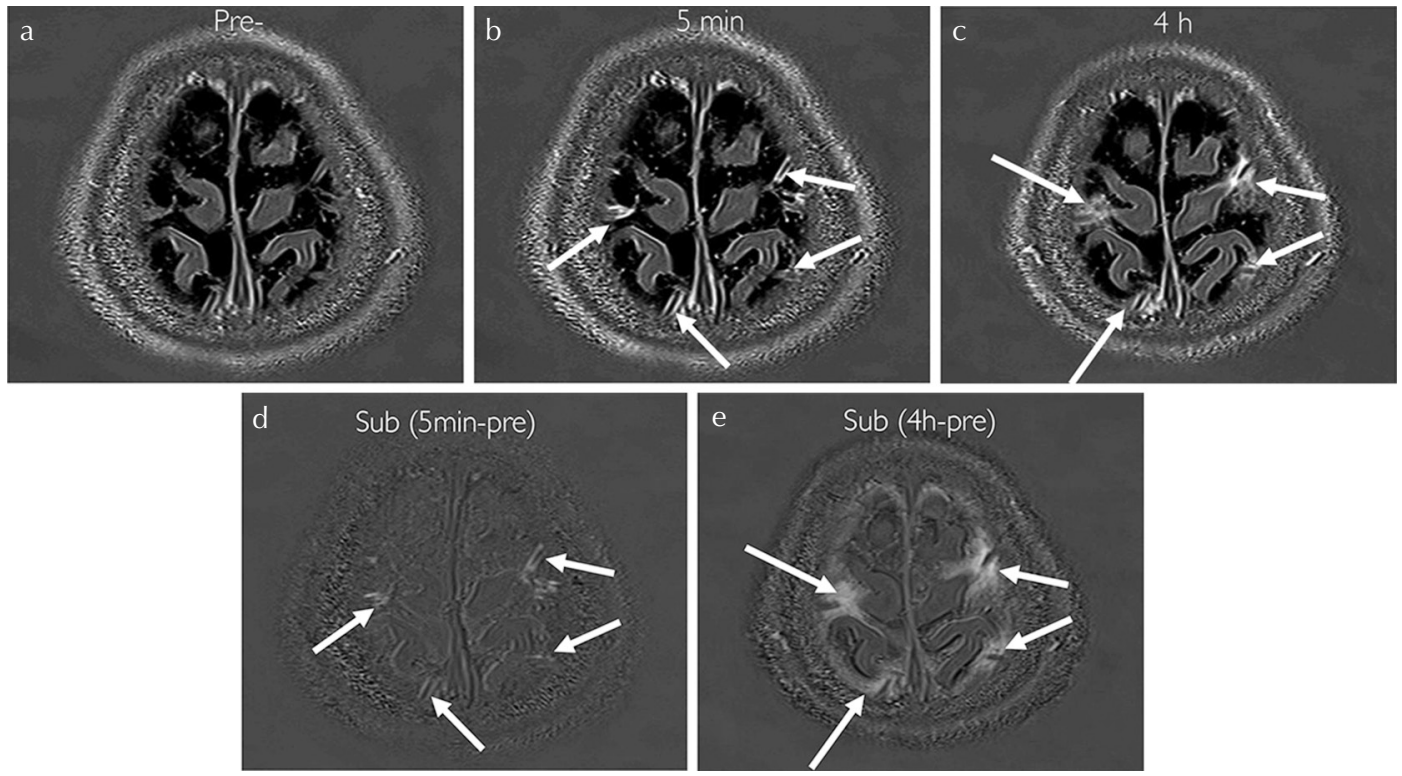

Fig. 1 A 57-year-old woman with a suspicion of endolymphatic hydrops. Three dimensional real inversion recovery (3D-real IR) images obtained at pre- (a), $5 \mathrm{~min}$ after (b) and $4 \mathrm{~h}$ after intravenous administration of single dose gadolinium-based contrast agent (IV-SD-GBCA) (c). (d) Subtracted image (b-a). (e) Subtracted image (c-a). At 5 min after IV-SD-GBCA, the peri-venous enhancement appears along the cortical veins (arrows in $\mathbf{b}-\mathbf{d}$ ). The areas of enhancement spread into the surrounding cerebrospinal fluid (CSF) space (arrows in $\mathbf{c}$ and $\mathbf{e}$ ). 

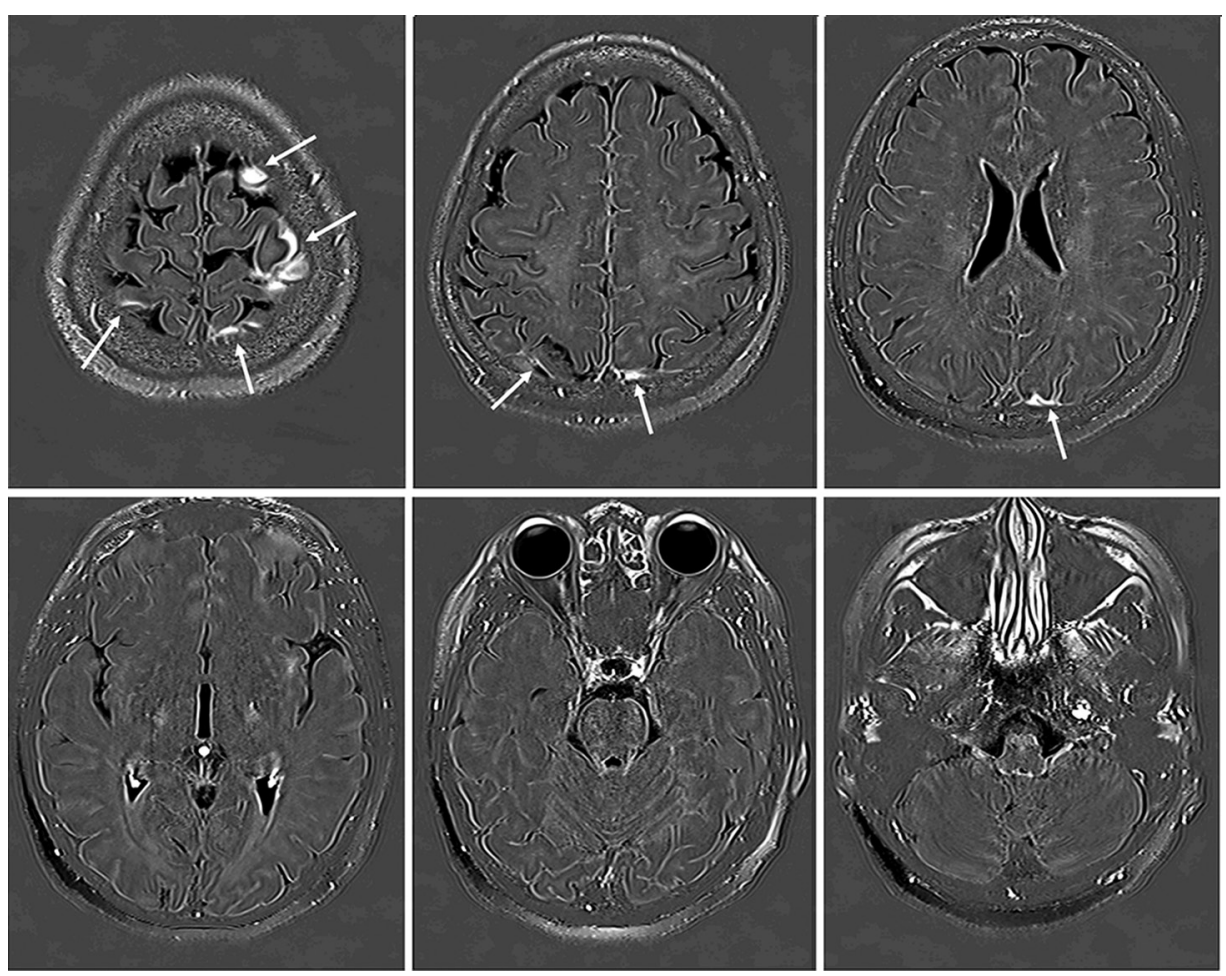

Fig. 2 A 49-year-old woman with a suspicion of endolymphatic hydrops. In the second part of this study, six out of 256 axial slices obtained at $4 \mathrm{~h}$ after intravenous administration of single dose gadolinium based contrast agent (IV-SD-GBCA) were selected at $20 \mathrm{~mm}$ increments from the vertex to the skull base for the image evaluation. The cerebrospinal fluid (CSF) around the cortical veins has a high signal intensity on Three dimensional real inversion recovery (3D-real IR)images (arrows).
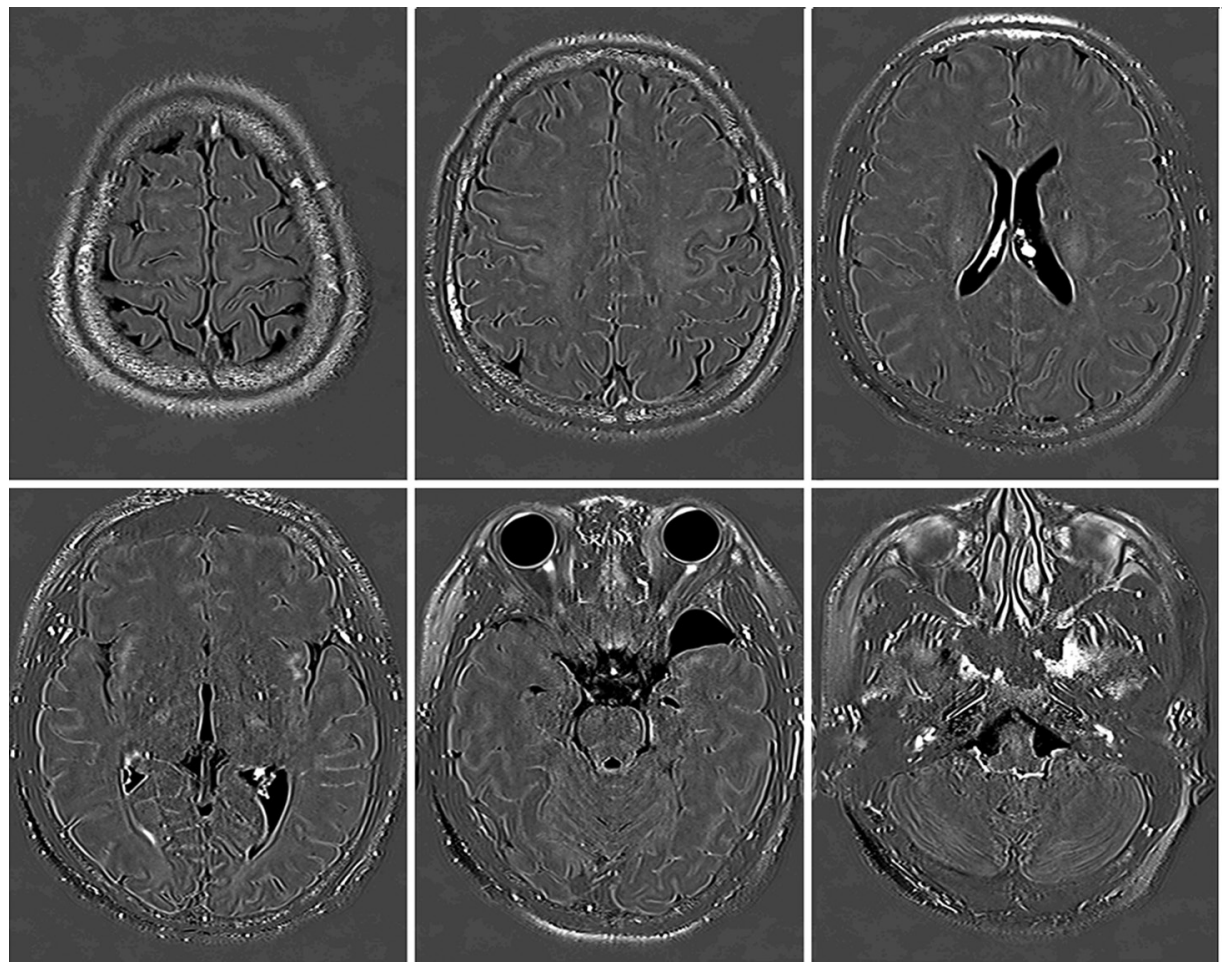

Fig. 3 A 17-year-old woman with a suspicion of endolymphatic hydrops. These images were obtained at $4 \mathrm{~h}$ after intravenous administration of single dose gadolinium based contrast agent (IV-SD-GBCA). Enhancement in the cerebrospinal fluid (CSF) around the cortical veins was not observed in this young patient.

have been suggested. ${ }^{1,9,14,21}$ The results of this present study add the cortical veins to this list of potential leaky points. It is important to know how GBCA in the CSF enters the brain parenchyma to better understand the mechanism for gadolinium deposition in the brain. ${ }^{3-5,8,22}$
The glymphatic system has been proposed recently as the waste clearance mechanism in the brain, which lacks a lymphatic system. ${ }^{6,7,23,24}$ In this proposed concept, the CSF enters the brain parenchyma through the peri-arterial space through aquaporin-4 channels in the end-feet of astrocytes. 
Subsequently, the CSF disperses into the interstitial spaces of the brain through the peri-venous spaces before circulating back into the CSF space, thereby clearing wastes such as aggregated proteins from the brain. Recently, there have been hot debates regarding this glymphatic concept. One study proposed a modification of the glymphatic concept. ${ }^{25}$ From the results of their animal experiments using intrathecal administration of superparamagnetic iron oxide, they speculated that some part of the waste in the peri-venous efflux route of glymphatic system might be absorbed through the venous wall into the venous lumen. This new concept postulates some permeability of the venous wall, which is also supported by the results of the present study. Also, it has been reported that the wall of leptomeningeal venule allows the transfer of antigen presenting cells. ${ }^{26}$ During the process of cell transfer, the wall of leptomeningeal venule might permit the passage of some molecules such as GBCA simultaneously. However, it is still unknown if the larger venous

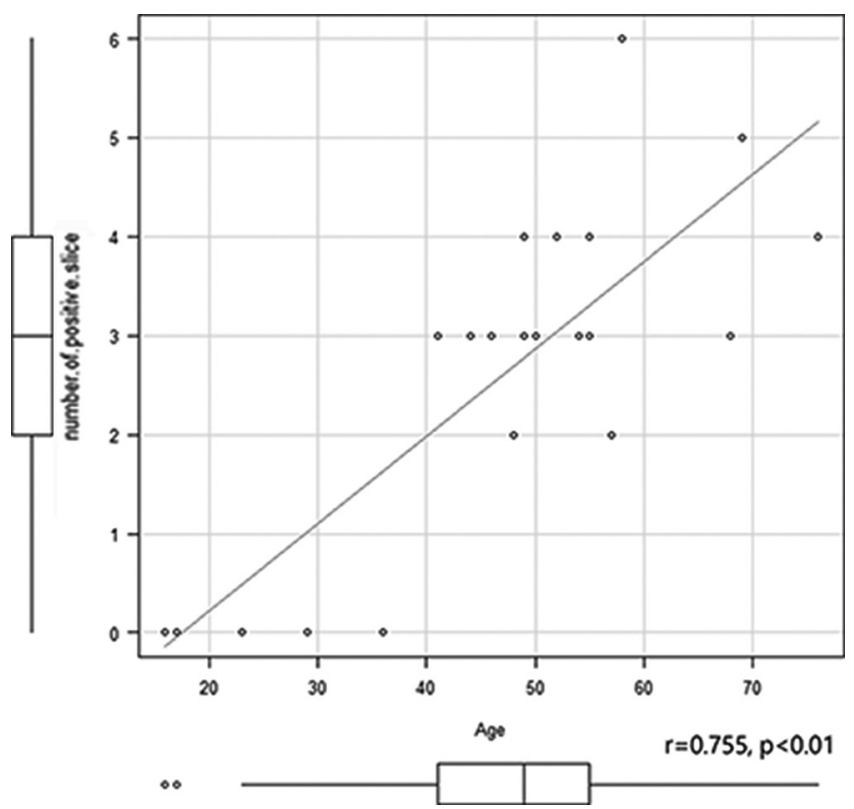

Fig. 4 Scatter plots of the correlations between the number of positive slices and age. There is a significant positive correlation between the number of slices with a positive enhancement and age. The Spearman's rank correlation coefficient was 0.755 $(P<0.01)$. Note that the gadolinium based contrast agent $(\mathrm{GBCA})$ leakage from the cortical veins into the surrounding cerebrospinal fluid (CSF) was positive in all elderly patients (37 years and above), but negative in all five younger patients (under 37 years). IV-SD-GBCA, intravenous administration of single dose gadolinium based contrast agent; CSF, cerebrospinal fluid. wall permits to pass the antigen presenting cells. Anatomically, it is reported that the pial sheath surrounding the venous wall is sparse, especially in the perivascular space. ${ }^{27}$ It is unknown whether the pial sheath surrounds the venous wall completely in the subarachnoid space, especially in aged subjects. Recently, it has been reported that the pial sheath in the subarachnoid space has pores or stomata, which allows the passage of antibodies. ${ }^{28}$ Considering these anatomical features, it is likely that GBCA molecules permeate from the cortical veins to the subarachnoid space.

In the present study, the leakiness of the cortical vein correlated significantly with aging. It has been reported that the focal peri-cortical enhancement on 3D-FLAIR MRI obtained at 16 min after IV-SD-GBCA was observed with normal aging and did not correlate with cognitive function. ${ }^{29}$ Although the images did not show directly that the leakage of GBCA comes from the cortical venous wall, they speculated that the peri-cortical CSF signal enhancement likely resulted from leakage of GBCA through the cortical or leptomeningeal vessels and, as such, represents a marker for focal loss of BBB integrity. ${ }^{29}$ They also speculated that the peri-cortical enhancement was likely not specific for a particular disease and also occurs in the context of normal aging. ${ }^{29}$ Using regular contrast 3D-FLAIR imaging and a shorter waiting time of $16 \mathrm{~min}$, their positive ratio of peri-cortical enhancement was only $28 \%$ even in a cohort at the age of 55 years and older. In the present study, we confirmed that the leak occurred in the region of the cortical veins by serially obtained images, and the increased signal intensity indicating a leak occurred in $100 \%$ of subjects that were 37 years or older. This increased signal intensity might be because of a longer interval after IV-GBCA and the higher sensitivity of the pulse sequence to low concentrations of GBCA in the CSF. Taken together, these studies suggest that chronic hypoxic and/or inflammatory processes in the cortex and leptomeninges during the aging process may underlie this neuroimaging phenomenon. However, further study is warranted to elucidate the clinical significance of the cortical venous leakiness in the various diseases using our sensitive method. To our knowledge, there is no histological study to show the apparent change of venous wall before and after the age around 37 years.

Recently, it has been reported that the signal enhancement of the perivascular space in the basal ganglia was negatively correlated to the volume of the cochlear in EH. ${ }^{14}$ In the present study, the leakiness of the cortical veins did not show a significant correlation with the degree of EH. It would be

Table 2 Number of patients with positive enhancement at each slice location

\begin{tabular}{lccccc}
\hline Slice location (vertical > basal) & Slice 1 & Slice 2 & Slice 3 & Slice 4 & Slice 5 \\
\hline $\begin{array}{l}\text { Number of patients with positive } \\
\text { enhancement out of } 21\end{array}$ & $16^{*}, * *$ & $13^{* * *}$ & $6^{*}$ & 9 & 9 \\
\begin{tabular}{l} 
Ratio of positive patients \\
\hline
\end{tabular} & 0.76 & 0.62 & 0.29 & 0.43 & 0.43 \\
\hline
\end{tabular}

$*, * *, * * *$, Significant difference. 
interesting to investigate the potential relationship between the leakiness of the cortical veins and disease processes other than EH. The new finding indicated in this present study might provide insight into the research in neuroimaging. Further study is warranted to elucidate the difference of GBCA leakage to CSF by aging and meningitis.

There are some limitations in this study. First, we did not include healthy volunteers. All subjects had a suspicion of EH. Although the degree of EH did not significantly correlate with the leakiness of the cortical veins, it is necessary to include healthy volunteers to completely rule out the effect of $\mathrm{EH}$ on the leakiness of the cortical veins. Second, the number of subjects is small. In particular, additional younger subjects should be included in the future study. Third, the lack of histopathological analysis of the leaky cortical veins prevents us from determining if the leakiness is from the venous wall itself or the surrounding vasa vasorum.

\section{Conclusion}

Intravenously administered GBCA from the cortical veins into the surrounding CSF. The leakiness of the cortical veins correlates significantly with age, but not with gender or degree of $\mathrm{EH}$.

\section{Conflicts of Interest}

The authors declare that they have no conflicts of interest.

\section{References}

1. Naganawa S, Suzuki K, Yamazaki M, Sakurai Y. Serial scans in healthy volunteers following intravenous administration of gadoteridol: time course of contrast enhancement in various cranial fluid spaces. Magn Reson Med Sci 2014; 13:7-13.

2. Naganawa S, Yamazaki M, Kawai H, Sone M, Nakashima T. Contrast enhancement of the anterior eye segment and subarachnoid space: detection in the normal state by heavily T2-weighted 3D FLAIR. Magn Reson Med Sci 2011; 10:193-199.

3. Li L, Gao FQ, Zhang B, Luo BN, Yang ZY, Zhao J. Overdosage of intrathecal gadolinium and neurological response. Clin Radiol 2008; 63:1063-1068.

4. Ringstad G, Vatnehol SAS, Eide PK. Glymphatic MRI in idiopathic normal pressure hydrocephalus. Brain 2017; 140:2691-2705.

5. Eide PK, Ringstad G. MRI with intrathecal MRI gadolinium contrast medium administration: a possible method to assess glymphatic function in human brain. Acta Radiol Open 2015; 4:2058460115609635.

6. Jost G, Frenzel T, Lohrke J, Lenhard DC, Naganawa S, Pietsch $\mathrm{H}$. Penetration and distribution of gadoliniumbased contrast agents into the cerebrospinal fluid in healthy rats: a potential pathway of entry into the brain tissue. Eur Radiol 2017; 27:2877-2885.
7. Iliff JJ, Lee H, Yu M, et al. Brain-wide pathway for waste clearance captured by contrast-enhanced MRI. J Clin Invest 2013; 123:1299-1309.

8. Gaberel T, Gakuba C, Goulay R, et al. Impaired glymphatic perfusion after strokes revealed by contrast-enhanced MRI: a new target for fibrinolysis? Stroke 2014; 45: 3092-3096.

9. Naganawa S, Taoka T, Kawai H, Yamazaki M, Suzuki K. Appearance of the organum vasculosum of the lamina terminalis on contrast-enhanced MR imaging. Magn Reson Med Sci 2018; 17:132-137.

10. Naganawa S, Kawai H, Sone M, Nakashima T. Increased sensitivity to low concentration gadolinium contrast by optimized heavily T2-weighted 3D-FLAIR to visualize endolymphatic space. Magn Reson Med Sci 2010; 9: 73-80.

11. Naganawa $S$, Kawai $H$, Taoka $T$, Sone M. Improved HYDROPS: imaging of endolymphatic hydrops after intravenous administration of gadolinium. Magn Reson Med Sci 2017; 16:357-361.

12. Naganawa S, Yamazaki M, Kawai H, Bokura K, Sone M, Nakashima T. Visualization of endolymphatic hydrops in Ménière's disease with single-dose intravenous gadoliniumbased contrast media using heavily $\mathrm{T}_{2}$-weighted 3D-FLAIR. Magn Reson Med Sci 2010; 9:237-242.

13. Nakashima T, Pyykkö I, Arroll MA, et al. Meniere's disease. Nat Rev Dis Primers 2016; 2:16028.

14. Ohashi T, Naganawa S, Katagiri T, Kuno K. Relationship between contrast enhancement of the perivascular space in the basal ganglia and endolymphatic volume ratio. Magn Reson Med Sci 2018; 17:67-72.

15. Okazaki Y, Yoshida T, Sugimoto $S$, et al. Significance of endolymphatic hydrops in ears with unilateral sensorineural hearing loss. Otol Neurotol 2017; 38:1076-1080.

16. Naganawa S, Satake H, Kawamura M, Fukatsu H, Sone M, Nakashima T. Separate visualization of endolymphatic space, perilymphatic space and bone by a single pulse sequence; 3D-inversion recovery imaging utilizing real reconstruction after intratympanic Gd-DTPA administration at 3 Tesla. Eur Radiol 2008; 18:920-924.

17. Naganawa S, Sone M, Yamazaki M, Kawai H, Nakashima T. Visualization of endolymphatic hydrops after intratympanic injection of Gd-DTPA: comparison of 2D and 3D real inversion recovery imaging. Magn Reson Med Sci 2011; 10:101-106.

18. Naganawa S, Kawai H, Taoka T, Sone M. Improved 3D-real inversion recovery: a robust imaging technique for endolymphatic hydrops after intravenous administration of gadolinium. Magn Reson Med Sci 2018 March 07 doi. org/10.2463/mrms.bc.2017-0158. [Epub ahead of print].

19. Nakashima T, Naganawa S, Pyykkö I, et al. Grading of endolymphatic hydrops using magnetic resonance imaging. Acta Otolaryngol Suppl 2009; 129:5-8.

20. Naganawa S, Nakane T, Kawai H, Taoka T. Lack of contrast enhancement in a giant perivascular space of the basal ganglion on delayed FLAIR images: implications for the glymphatic system. Magn Reson Med Sci 2017; 16:89-90.

21. Naganawa S, Nakane T, Kawai H, Taoka T. Gd-based contrast enhancement of the perivascular spaces in the basal ganglia. Magn Reson Med Sci 2017; 16:61-65. 
22. Lee H, Mortensen K, Sanggaard S, et al. Quantitative GdDOTA uptake from cerebrospinal fluid into rat brain using 3D VFA-SPGR at 9.4T. Magn Reson Med 2018; 79:1568-1578.

23. Iliff JJ, Chen MJ, Plog BA, et al. Impairment of glymphatic pathway function promotes tau pathology after traumatic brain injury. J Neurosci 2014; 34:16180-16193.

24. Jessen NA, Munk AS, Lundgaard I, Nedergaard M. The glymphatic system: a beginner's guide. Neurochem Res 2015; 40:2583-2599.

25. Shen $Y$, Jiang Q, Ding G, Guys N, Haacke E, Hu J. Investigation of the role of the venous system and the glymphatic system in brain waste clearance. ISMRM 25th Annual Meeting \& Exhibition, Honolulu 2017;0590.

26. Engelhardt B, Carare RO, Bechmann I, Flügel A, Laman JD, Weller RO. Vascular, glial, and lymphatic immune gateways of the central nervous system. Acta Neuropathol 2016; 132:317-338.

27. Zhang ET, Inman CB, Weller RO. Interrelationships of the pia mater and the perivascular (Virchow-Robin) spaces in the human cerebrum. J Anat 1990; 170: 111-123.

28. Pizzo ME, Wolak DJ, Kumar NN, et al. Intrathecal antibody distribution in the rat brain: surface diffusion, perivascular transport and osmotic enhancement of delivery. J Physiol (Lond) 2018; 596:445-475.

29. Freeze WM, Schnerr RS, Palm WM, et al. Pericortical enhancement on delayed postgadolinium fluid-attenuated inversion recovery images in normal aging, mild cognitive impairment, and Alzheimer disease. AJNR Am J Neuroradiol 2017; 38:1742-1747. 